\title{
Tabular Organisation of Ionothermally Prepared MOFs To Extrapolate Chemical Trends and Successfully Predict Synthesis Results
}

\author{
Hyun Chang $\mathrm{Oh}^{1,2}$, Sukwoo Jung ${ }^{1}$, Ilju Ko ${ }^{1,2}$ and Eun Young Choi*1 \\ ${ }^{1}$ Korea Science Academy of KAIST, Korea \\ ${ }^{2}$ Korea Advanced Institute of Science and Technology, Korea
}

Received: April 13, 2018; Published: April 24, 2018

*Corresponding author: Eun Young Choi, Korea Science Academy of KAIST, 111 Baegyanggwanmun-ro, Busanjin-gu, Busan 614-100 Korea, Tel: 821020126757; Email: faujasite@kaist.ac.kr

\section{Introduction}

Metal organic frameworks, or MOFs, have a strength over other classes of materials in which the constituents of the frameworks may be chosen among the vast range of organic ligands and metals, and such combination leads to nearly infinite possibility of novel frameworks. This freedom of choices expands when the variety of synthesis methods for MOFs is considered. Ionothermal synthesis, which makes use of ionic liquids for both the purpose of solvent and template, is a relatively recently introduced method that best exercises such potential on its own [1], along with many advantages like safety, which is associated with the low vapour pressure of ionic liquids [2], thermal stability [3], and safety in environmental means relative to its organic predecessors [4]. The cation and anion that comprises the ionic solvent used in the method may be easily substituted with their close chemical relatives, chloride with another halide for example, and finely tune the reaction environment.

The series of ionic liquids (RMI-X) composed of 1-alkyl-3methylimidazolium (RMI) as cations and halide (X) as anions is one of the solvents most extensively employed for such purposes, due to the ease of modifying the length of the alkyl chain of the cations and varying the anion along the halide column. Research under various circumstances is prerequisite to the full practice of the unique advantages of ionothermal synthesis, but regardless of however many frameworks reported, it accompanies the need to make the effort to discover the chemical trends appearing on the arrangement of the reports under the criteria of the piecemeal difference in the reaction environment that brought about the difference in structure. The ionothermal synthesis with RMI-X has produced a system of frameworks with benzene tricarboxylic acid (BTC) as the organic linker and nickel [5], cadmium [6], manganese [7], and cobalt [8] being the metal component.
In this work, these frameworks have been organised in tables with the two axes of the length of the alkyl side chain of the cation and the type of the halide anion. The structures on the tables were then analysed to identify groups of topological similarities, which unveiled sequential chemical trends. Similarly, to a previous report with the cobalt-BTC system [9], the structures in the nickel-BTC system were analysed to design expectations for the combinations on the table that were yet to be reported. The extrapolations were then shown to be valid with powder X-ray diffraction (PXRD) analysis of the compounds prepared.

\section{Results and Discussion}

In a previous study, structures were prepared by the ionothermal reaction of $\mathrm{Ni}^{2+}$ and $\mathrm{H}_{3} \mathrm{BTC}$ in the ionic liquids with cation and halide combinations, ranging from $\mathrm{Cl}^{-}$to $\mathrm{I}^{-}$in the anions, and ethyl- to butyl- in the alkyl chain length of the RMI cations [10]. The factors that lead each combination to the different fate of structure A and B were explained, including the differing nucleophilicity and/or basicity of halide ions favouring the two different protonated states of the BTC ligand. The power of such reasoning is powerful in that it provides the logical background for predicting the results for similar conditions-a point where guessing becomes anticipating. In the frameworks prepared with [PMI] and [BMI] in the Ni-BTC system, the structure shifted from A class to B class with a larger pore size as the halides of IL increased in size. The shift was not observed in [EMI], which is probably too small to induce the change even with the largest anion, iodide.

The size of the cation also influenced the shifting point in [PMI] and [BMI] rows; the shift occurred between bromide and iodide with [PMI] while it occurred between smaller anions, chloride and bromide. Although not reported in preceding research, further increasing the cation size is expected to bring the shifting point 
even more leftward, possibly to a point where A structure is no longer observed. The next question to be addressed is whether the pores of B structure are large enough to incorporate the elongated cations. If not, the system will either prefer shifting to another class of structure with larger pores or fail to form crystals if such structure is unstable. In beforehand, the frameworks of A and B structures were compared and analysed whether they could incorporate cations of longer side chains. Figure 1 shows that the pores of B structure have the shape of straight linear channels while A structure has non-straight channels.

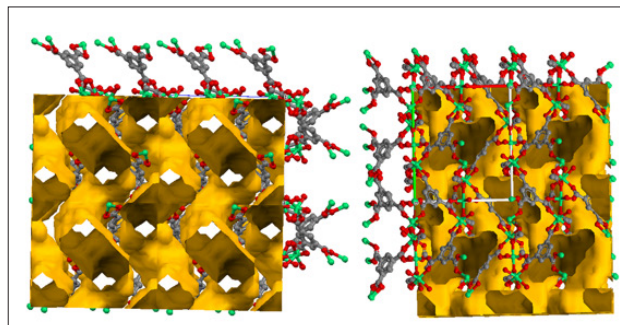

(a)

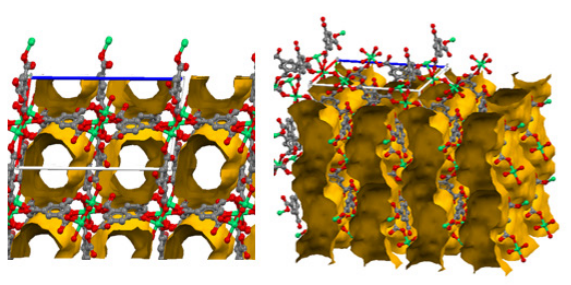

(b)

Figure 1: The framework of $[\mathrm{BMI}]_{2}\left[\mathrm{Ni}_{3}(\mathrm{BTC})_{2}(\mathrm{OAc})_{2}\right]$ (A type) and $[\mathrm{BMI}]_{2}\left[\mathrm{Ni}_{3}(\mathrm{HBTC})_{4}\left(\mathrm{H}_{2} \mathrm{O}\right)_{2}\right]$ (B type) in Ni-BTC system. B structure have the shape of straight linear channels while A structure has non-straight channels. BMI cations are omitted for clarity.

It becomes evident that longer cations will conflict with the bumps of channels in A structure and thus exert a chemical pressure toward B structure. The straight B channels are shown to be capable of housing cations of theoretically any length, though it may be possible only in lesser molar quantities. Overall, we expect B frameworks to be stable with PEMI and HMI incorporated in their pores. Powder X-ray diffraction of the prepared frameworks for the PEMI and HMI row coincided well enough with the data of reported B structures, which supports the validity of our expectations (Figure 2). Systems in which nickel component was substituted with cadmium and manganese showed trends in a similar sequential manner and were illustrated in Tables 1-3 respectively.
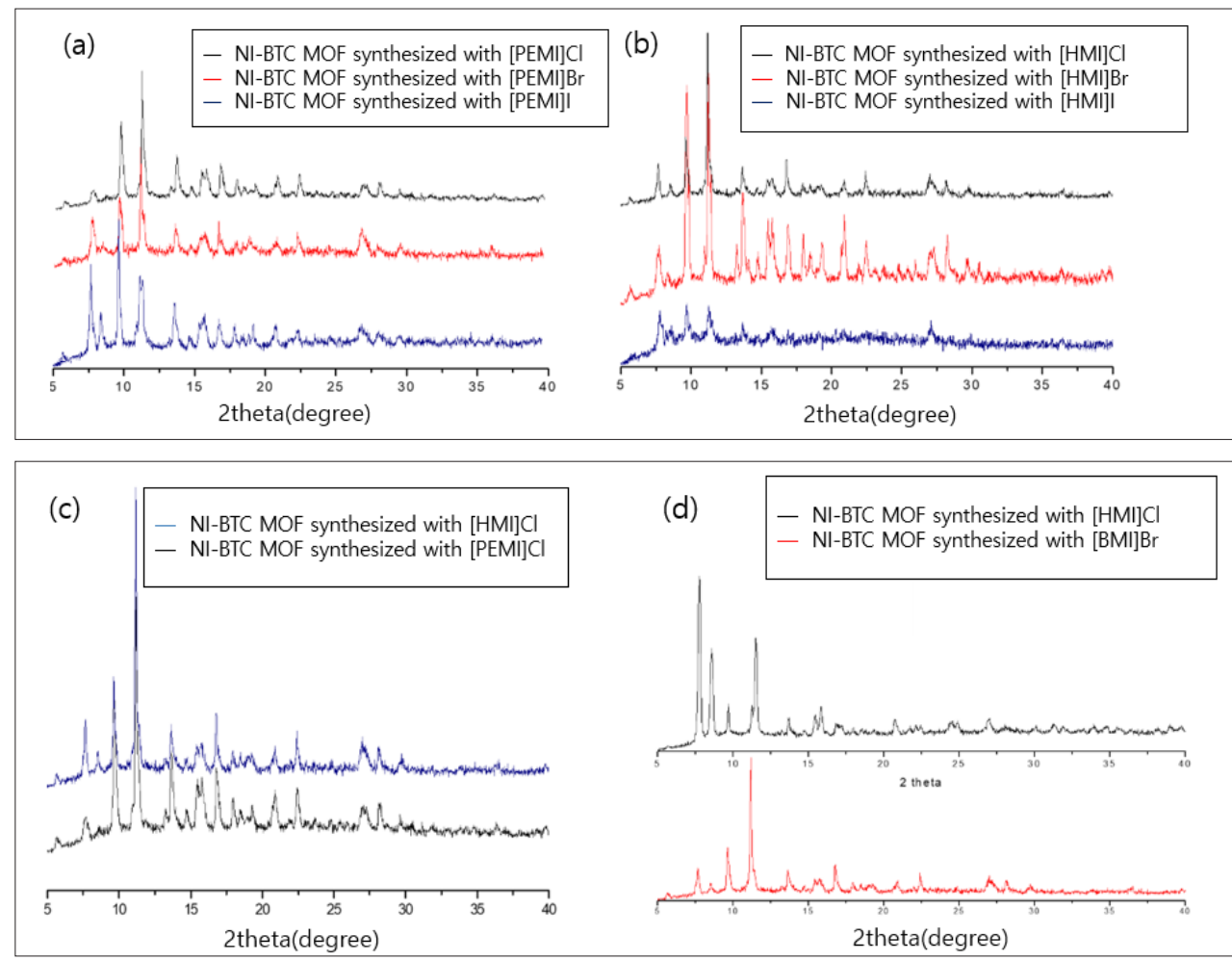

Figure 2: Comparison of PXRD data of Ni-BTC MOFs synthesized with a) [PEMI]X, where $\mathrm{X}$ is $\mathrm{Cl}, \mathrm{Br}, \mathrm{I}, \mathrm{b})[\mathrm{HMI}] \mathrm{X}, \mathrm{where} \mathrm{X}$ is $\mathrm{Cl}, \mathrm{Br}, \mathrm{I}, \mathrm{c})[\mathrm{HMI}] \mathrm{Cl}$ and $[\mathrm{PEMI}] \mathrm{Cl}, \mathrm{d})[\mathrm{HMI}] \mathrm{Cl}$ and $[\mathrm{BMI}] \mathrm{Br}$. Comparison within the newly synthesized [PEMI] and [HMI] rows show the major peaks coinciding with each other, suggesting that they have the same structure (a,b,andc). Comparison of the MOF synthesized with [HMI]Cl with the reported MOFs prepared with [BMI]Br shows both MOFs have similar framework, which let us identify the [PEMI] and [HMI] MOF groups in the B class. 
Table 1: Organisation of structures in Nickel-BTC system on the length of the alkyl side chain of the cation and the halide anion. Structures sharing a topological identity were labelled under the same alphabet, while the numbers denote minor difference among them. Each labels denote, $[\mathrm{RMI}]_{2}\left[\mathrm{Ni}_{3}(\mathrm{BTC})_{2}(\mathrm{OAc})_{2}\right]\left(\mathrm{RMI}=\mathrm{EMI}\right.$ for A1, PMI for A2, BMI for A3), $\left[\mathrm{RMI}_{2}\left[\mathrm{Ni}_{3}(\mathrm{HBTC})_{4}\left(\mathrm{H}_{2} \mathrm{O}\right)_{2}\right](\mathrm{RMI}=\right.$ BMI for B2, PEMI forB3, HMI for B4). A1, A2, A3, B1, B2 reported in [10]. B3 and B4 were first reported in this work.

\begin{tabular}{|c|c|c|c|}
\hline $\mathbf{N i}(\mathbf{O A c})_{2}-\mathbf{H}_{3} \mathbf{B T C}$ & CI & Br & I \\
\hline$[\mathrm{EMI}]$ & A1 & A1 & A1 \\
\hline$[\mathrm{PMI}]$ & A2 & A2 & B2 \\
\hline$[\mathrm{BMI}]$ & A3 & B3 & B3 \\
\hline$[\mathrm{PEMI}]$ & B3 & B4 & B4 \\
\hline$[\mathrm{HMI}]$ & B4 & & B \\
\hline
\end{tabular}

Table 2: Organisation of structures in Cadmium-BTC system on the length of the alkyl side chain of the cation and the halide anion. Structures sharing a topological identity were labelled under the same alphabet, while the numbers denote minor difference among them. Each labels denote, a1-[EMI][Cd $\left.{ }_{2}(B T C) C_{2}\right]$ for a1, [RMI][Cd(BTC)] (RMI for b1, PMI for b2). Combinations that have not been reported were left blank. All structures in this table were reported in [6].

\begin{tabular}{|c|c|c|c|}
\hline $\mathbf{C d}\left(\mathrm{NO}_{3}\right)_{2}-\mathbf{H}_{3} \mathbf{B T C}$ & CI & Br & I \\
\hline$[\mathrm{EMI}]$ & $\mathrm{a} 1$ & $\mathrm{~b} 1$ & $\mathrm{~b} 1$ \\
\hline$[\mathrm{PMI}]$ & $\mathrm{b} 2$ & $\mathrm{~b} 2$ & $\mathrm{~b} 2$ \\
\hline$[\mathrm{BMI}]$ & & & \\
\hline
\end{tabular}

Table 3: Organisation of structures in Manganese-BTC system on the length of the alkyl side chain of the cation and the halide anion. Structures sharing a topological identity were labelled under the same alphabet, while the numbers denote minor difference among them. Each labels denote, EMI][Mn(BTC)] for a1, [PMI][Mn(BTC)] for a2, [PMI][Mn(BTC)] for a3. Combinations that have not been reported were left blank. All structures in this table were reported in [7].

\begin{tabular}{|c|c|c|c|}
\hline $\mathbf{M n}(\mathbf{O A c})_{2}-\mathbf{H}_{3} \mathbf{B T C}$ & CI & Br & I \\
\hline$[\mathrm{EMI}]$ & $\alpha 1$ & $\alpha 1$ & $\alpha 1$ \\
\hline$[\mathrm{PMI}]$ & $\alpha 2$ & $\alpha 2$ & $\alpha 3$ \\
\hline$[\mathrm{BMI}]$ & & & \\
\hline$[\mathrm{PMI}]$ & & & \\
\hline
\end{tabular}

\section{Conclusion}

We have explored the chemical trend observed in the Ni-BTC MOF system and explained it in the context of cation size and pore shape. Analysing the pore in B frameworks has shown that it is straight linear and suggested the capability of the structure to incorporate larger cations. Crystals have been prepared with [PEMI] and [HMI], which have longer side chains, and their powder X-ray diffraction patterns were compared to the reported MOFs with B structure, which supported that they share a common structure. Tabular organisation of Ionothermally prepared MOFs based on the cation and anions of IL is useful in a way that chemical trend becomes more evident and may be used to extrapolate the outcomes of untried combinations.

\section{References}

1. Parnham ER, Morris RE (2007) Ionothermal synthesis of zeolites, metal-organic frameworks, and inorganic-organic hybrids. Accounts of Chemical Research 40(10): 1005-1013.

2. Earle MJ, Esperanc JM, MA Gilea, Lopes JN, Rebelo LP, et al. (2006) The distillation and volatility of ionic liquids. Nature 439(7078): 831-834.

3. HL Ngo, Le Compte K, Hargens L, McEwen AB (2000) Thermal properties of imidazolium ionic liquids. Thermochimica Acta357-358: 97-102.

4. Rogers RD, Seddon KR (2003) Ionic liquids-solvents of the future?. Science 302(5646): 792-793.
5. Xu L, Choi EY, Kwon YU (2008) A new 3D nickel(II) framework composed of large rings: Ionothermal synthesis and crystal structure. Journal of Solid State Chemistry 181(11): 3185-3188.

6. Xu L, Choi EY, Kwon YU (2008) Combination effects of cation and anion of ionic liquids on the cadmium metal-organic frameworks in ionothermal systems. Inorganic Chemistry 47(6): 1907-1909.

7. Ling Xu, Young Kwon Uk, Baltazar de Castro, and Luís Cunha-Silva (2013) Novel Mn(II)-Based Metal-Organic Frameworks Isolated in Ionic Liquids. Crystal Growth \& Design 13(3): 1260-1266.

8. Wang YL, N Zhang, Liu QY, Qing Yan Liu, Xin Yang, et al. (2011) Ionothermal syntheses and crystal structures of two cobalt(II)carboxylate compounds with different topology. Inorganic Chemistry Communications 14(2): 380-383.

9. Il Ju Ko, Hyun-Chang Oh, Yong-Jun Cha, Chae Hyeok Han, EunYoung Choi (2017) Ionothermal Synthesis of a Novel 3D Cobalt Coordination Polymer with a Uniquely Reported Framework: $[\mathrm{BMI}]_{2}\left[\mathrm{Co}_{2}(\mathrm{BTC})_{2}(\mathrm{H} 2 \mathrm{O})_{2}\right]$. Advances in Materials Science and Engineering 2017(28): 1-6.

10. Ling Xu, Shihai Yan, Eun-Young Choi, Jin Yong Lee, Young Kwon Uk (2009) Product Control by Halide Ions of Ionic Liquids in the Ionothermal Syntheses of Ni-(H)BTC Metal-Organic Frameworks. Chemical Communications 23(30): 3431-3433. 


\section{(C) (i) This work is licensed under Creative}

Submission Link: https://biomedres.us/submit-manuscript.php

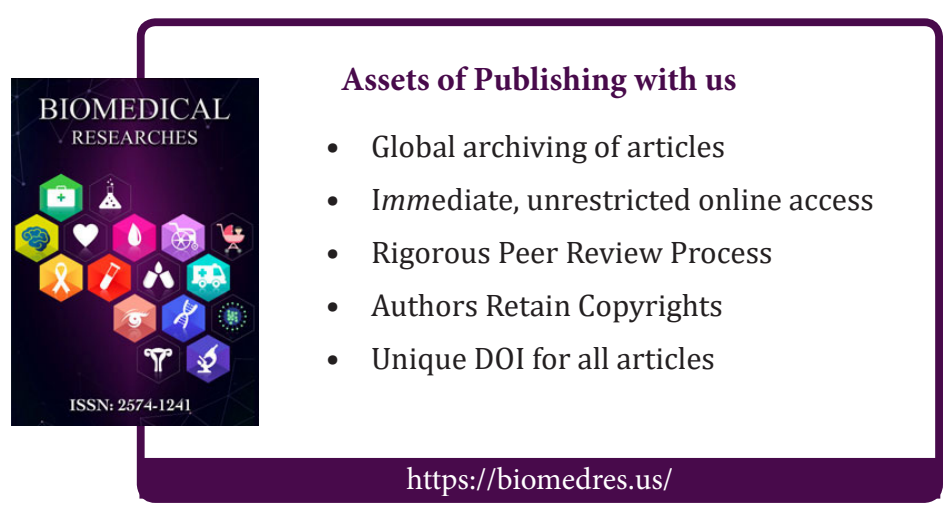

